\title{
Single Cell Gel Electrophoresis
}

National Cancer Institute

\section{Source}

National Cancer Institute. Single Cell Gel Electrophoresis. NCI Thesaurus. Code C96515.

A rapid, simple, visual and sensitive technique for measuring DNA breakage in individual mammalian cells in response to a test agent. A cell is immobilized in agarose and made permeable and the broken DNA migrates out under the influence of the electric field. A damaged cell has the appearance of a comet with a brightly fluorescent cell body and a tail whose length and fluorescence intensity are related to the number of DNA-strand breaks induced by the test agent. 